\section{Investigation of Two-Dimensional Unsteady Stagnation-Point Flow and Heat Transfer Impinging on an Accelerated Flat Plate}

\author{
Ali Shokrgozar Abbassi \\ Ph.D. Student
}

\author{
Asghar Baradaran Rahimi ${ }^{1}$ \\ Professor \\ e-mail: rahimiab@yahoo.com \\ Faculty of Engineering, \\ Ferdowsi University of Mashhad, \\ Mashhad 91775-1111, Iran
}

General formulation and solution of Navier-Stokes and energy equations are sought in the study of two-dimensional unsteady stagnation-point flow and heat transfer impinging on a flat plate when the plate is moving with variable velocity and acceleration toward main stream or away from it. As an application, among others, this accelerated plate can be assumed as a solidification front which is being formed with variable velocity. An external fluid, along z-direction, with strain rate a impinges on this flat plate and produces an unsteady two-dimensional flow in which the plate moves along z-direction with variable velocity and acceleration in general. A reduction of Navier-Stokes and energy equations is obtained by use of appropriate similarity transformations. Velocity and pressure profiles, boundary layer thickness, and surface stress-tensors along with temperature profiles are presented for different examples of impinging fluid strain rate, selected values of plate velocity, and Prandtl number parameter. [DOI: $10.1115 / 1.4005742]$

Keywords: stagnation-point flow and heat transfer, unsteady flow, viscous fluid, accelerated plate, similarity solution

\section{Introduction}

There are many solutions of the Navier-Stockes and energy equations regarding the problem of stagnation-point flow and heat transfer in the vicinity of a flat plate or a cylinder. These studies include: two-dimensional stagnation-point flow on a stationary flat plate [1]; and axisymmetric stagnation flow on a circular cylinder [2]. Further studies are: heat transfer in an axisymmetric stagnation flow on a cylinder [3]; nonsimilar axisymmetric stagnation flow on a moving cylinder [4]; unsteady viscous flow in the vicinity of an axisymmetric stagnation-point on a cylinder [5]; radial stagnation flow on a rotating cylinder with uniform transpiration [6]; axisymmetric stagnation flow toward a moving plate [7] in which the plate moves in its own plane. The more recent studies in this area are: oscillating stagnation-point flow [8]; axisymmetric stagnation-point flow and heat transfer of a viscous fluid on a moving cylinder with time-dependent axial velocity and uniform transpiration [9]; axisymmetric stagnation-point flow and heat transfer of a viscous fluid on a rotating cylinder with timedependent angular velocity and uniform transpiration [10]; similarity solution of unaxisymmetric heat transfer in stagnation-point flow on a cylinder with simultaneous axial and rotational move-

\footnotetext{
${ }^{1}$ Corresponding author.

Contributed by the Heat Transfer Division of ASME for publication in the Journal of Heat Transfer. Manuscript received August 26, 2010; final manuscript received November 17, 2011; published online April 26, 2012. Assoc. Editor: Darrell W. Pepper.
}

ments [11]; and recently, nonaxisymmetric three-dimensional stagnation-point flow and heat transfer on a flat plate [12], threedimensional stagnation flow and heat transfer on a flat plate with transpiration [13], and exact solution of three-dimensional unsteady stagnation flow on a heated plate [14]. Among all the studies above only in Ref. [8], the flat plate oscillates vertically and some particular solutions have been obtained by use of Fourier's expansions and in Ref. [14] which is an exact solution of a heated plate. Similarity solution of stagnation-point flow and heat transfer problem on a flat plate with arbitrary vertical movement is nonexisting in the literature.

In this study, the general two-dimensional unsteady viscous stagnation-point flow and heat transfer in the vicinity of a flat plate are investigated where this flat plate is moving toward or away from the impinging flow with variable velocity and acceleration. The Navier-Stokes equations along with energy equation are solved. One of the applications of this type of moving plate is encountered in solidification and melting problems. The external fluid, along $z$-direction, with strain rate $a$ impinges on a flat plate while the plate is moving with variable velocity and acceleration along $z$-direction. A similarity solution of the Navier-Stokes and energy equations is derived in this problem. The obtained ordinary differential equations are solved by using finite-difference numerical techniques. Velocity and pressure profiles, boundary layer thickness, and surface stress-tensors along with temperature profiles are presented for different examples of impinging fluid strain rate, selected values of plate velocity, and Prandtl number parameter.

\section{Problem Formulation}

Flow is considered in Cartesian coordinates $(x, z)$ with corresponding velocity components $(u, w)$, see Fig. 1 . We consider the laminar unsteady incompressible flow and heat transfer of a viscous fluid in the neighborhood of stagnation-point on a moving flat plate located in the $z=0$ plane at $t=0$. An external fluid, along $z$-direction, with strain rate $a$ impinges on this accelerated flat plate along $z$-direction and produces a two-dimensional flow on the plate. Obviously, in the situation of moving plate, the boundary layer thickness along $x$-direction changes in contrary to the case of fix plate when it is with constant thickness. As an application, this accelerated plate can be assumed as a solidification front which is moving with variable velocity along the $z$-axis in which the solid thickness is growing steadily in $x$ direction, [15].

The unsteady Navier-Stokes and energy equations in Cartesian coordinates governing the flow and heat transfer are given as

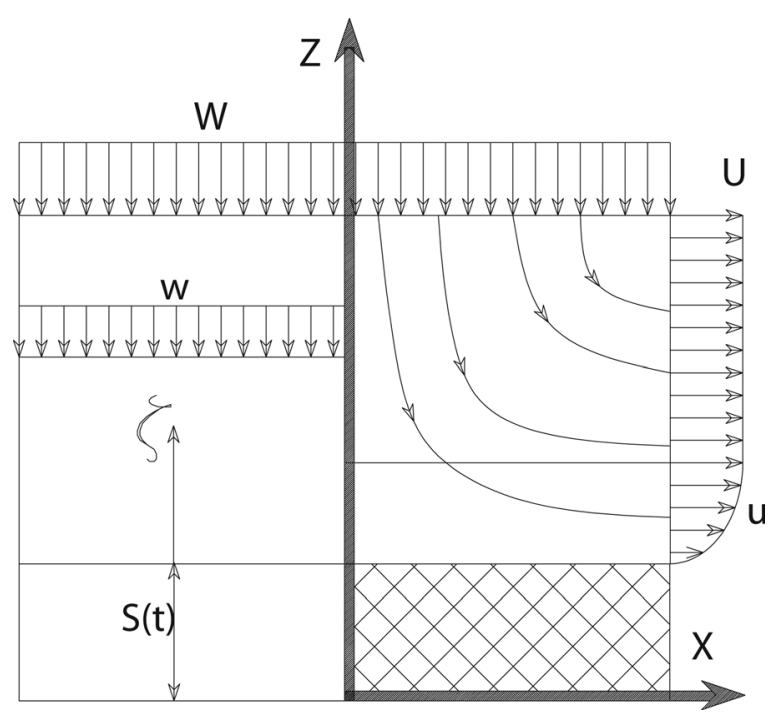

Fig. 1 Schematic problem graph 


$$
\begin{gathered}
\frac{\partial u}{\partial x}+\frac{\partial w}{\partial z}=0 \\
\frac{\partial u}{\partial t}+u \frac{\partial u}{\partial x}+w \frac{\partial u}{\partial z}=-\frac{1}{\rho} \frac{\partial P}{\partial x}+\nu\left[\frac{\partial^{2} u}{\partial x^{2}}+\frac{\partial^{2} u}{\partial z^{2}}\right] \\
\frac{\partial w}{\partial t}+u \frac{\partial w}{\partial x}+w \frac{\partial w}{\partial z}=-\frac{1}{\rho} \frac{\partial P}{\partial z}+\nu\left[\frac{\partial^{2} w}{\partial x^{2}}+\frac{\partial^{2} w}{\partial z^{2}}\right] \\
\frac{\partial T}{\partial t}+u \frac{\partial T}{\partial x}+w \frac{\partial T}{\partial z}=\alpha\left[\frac{\partial^{2} T}{\partial x^{2}}+\frac{\partial^{2} T}{\partial z^{2}}\right]
\end{gathered}
$$

where $P, \rho, \nu$, and $\alpha$ are the fluid pressure, density, kinematics viscosity, and thermal diffusivity.

\section{Similarity Solution}

3.1 Fluid Flow Solution. The classical potential flow solution of the governing Eqs. (1)-(3) is as follows:

$$
\begin{gathered}
U=a(t) x \\
W=-a(t) \zeta \\
P_{\infty}=P_{0}-\frac{1}{2} \rho V_{1(u, w)}^{2}-\rho \int_{\infty}^{2} \frac{\partial V}{\partial t} d r \\
=0-\rho \frac{a(t)^{2}}{2}\left(x^{2}+\zeta^{2}\right)-\rho \int \frac{\partial}{\partial t}\left[a(t) \sqrt{\left(x^{2}+\zeta^{2}\right)}\right] d r
\end{gathered}
$$

where $p_{0}$ is stagnation pressure, $V$ is total velocity, $r$ is the flow passage, $S(t)$ is the amount of plate displacement in z-direction, and $\zeta=(z-S(t))$. By use of Eq. (7) on a streamline one obtains potential pressure. The cross-section which is at infinity is inside the potential region at which the variation of plate displacement is felt by the external flow. Therefore, the flow strain rate changes according to the plate velocity and so it becomes a timedependent quantity $a(t)$. This is equivalent to the plate velocity being superimposed on the external flow, therefore $\zeta=(z-S(t))$.

A reduction of the Navier-Stokes equations is sought by the following coordinate separation in which the solution of the viscous problem inside the boundary layer is obtained by composing the inviscid and viscous parts of the velocity components as the following:

$$
\begin{gathered}
u=a(t) x f^{\prime}(\eta) \\
w=-\sqrt{\frac{v}{a_{0}}} a(t) f(\eta) \\
\eta=\sqrt{\frac{a_{0}}{v}}(z-S(t)), \quad \text { and } \quad \zeta=(z-S(t))
\end{gathered}
$$

where terms involving $f(\eta)$ in Eqs. (8) and (9) comprise the Cartesian similarity form for unsteady stagnation-point flow and prime denotes differentiation with respect to $\eta$. Note, boundary layer is defined here as the edge of the points where their velocity is $99 \%$ of their corresponding potential velocity. Transformations (8)-(10) satisfy (1) automatically and their insertion into Eqs. (2)-(3) yields an ordinary differential equation in terms of $f(\eta)$ and an expression for the pressure

$$
f^{\prime \prime \prime}+f^{\prime \prime}(\tilde{\dot{S}}(t)+\tilde{a}(t) f)-\tilde{a}(t) f^{\prime 2}-\frac{f^{\prime}}{\tilde{a}(t)} \frac{\partial \tilde{a}(t)}{\partial \tau}-\frac{1}{\tilde{a}(t) \xi} \frac{\partial \tilde{P}}{\partial \xi}=0
$$

in which

$$
\frac{1}{\tilde{a}(t) \xi} \frac{\partial \tilde{P}}{\partial \xi}=-\tilde{a}(t)-\left(\frac{1}{\tilde{a}(t)} \frac{\partial \tilde{a}(t)}{\partial \tau}-\frac{2 \eta \tilde{\dot{S}}}{\xi^{2}+\eta^{2}}+\frac{\tilde{\dot{S}}}{\eta}\right)\left(1-\frac{\eta^{2}}{\xi^{2}}\right)
$$

$$
\begin{gathered}
\frac{\partial \tilde{a}(t)}{\partial \tau}=\frac{-\tilde{a}_{0} \tilde{\dot{S}}}{\eta_{0}}+\frac{\tilde{\tilde{S}}}{\eta_{0}}+\frac{\tilde{\dot{S}}^{2}}{\eta_{0}^{2}} \\
\tilde{P}=-\tilde{a}(t)\left(f^{\prime}-1\right)-\tilde{a}(t) \tilde{\dot{S}}(f-f(\eta=\delta))+\tilde{a}^{2}(t) \frac{\left(f^{2}-f^{2}(\eta=\delta)\right)}{2} \\
-A \int_{\eta}^{\delta} f(\eta) d \eta-\frac{\tilde{a}^{2}(t)}{2}\left(\xi^{2}+\eta^{2}\right) \\
-\left[A\left(\frac{\xi^{2}+\eta^{2}}{2}\right)-2 \tilde{a}(t) \tilde{\dot{S}} \int_{\infty}^{2} \frac{\eta\left(\xi^{2}-\eta^{2}\right)}{\xi\left(\xi^{2}+\eta^{2}\right)} d \xi+\tilde{a}(t) \tilde{\dot{S}}\left(\frac{\xi^{2}}{3 \eta}+\eta\right)\right]_{\infty}^{\delta}
\end{gathered}
$$

where

$$
\begin{gathered}
\tilde{P}(x, z, t)=\frac{P(x, z, t)}{\rho a_{0} v}, \quad \tilde{S}(t)=\sqrt{\frac{a_{0}}{v}} S(t), \quad \tilde{a}(t)=\frac{a(t)}{a_{0}}, \\
\tilde{\dot{S}}(t)=\dot{S}(t) / \sqrt{a_{0} v}, \quad \xi=\sqrt{\frac{a_{0}}{v}} x
\end{gathered}
$$

in which, "dot" denotes differentiation with respect to $t$, and quantities $\tilde{P}(x, z, t), \tilde{a}(t), \tilde{S}(t), \tilde{\dot{S}}(t), \xi$ are nondimensional forms of quantities $P(x, z, t), a(t), S(t), \dot{S}(t)$, and $x$, respectively. Pressure variation with respect to $\xi$ is presented by Eq. (12) and strain variation with respect to time which is the plate acceleration is presented by Eq. (13). Relation (14) which represents pressure is obtained by integrating Eq. (3) in z-direction and by use of the potential flow solution (5)-(7) as boundary conditions. Here, Eq. (11) is in the most general form for any arbitrary flat plate vertical movement and the boundary conditions for this equation are

$$
\begin{gathered}
\eta=0: \quad f=0, \quad f^{\prime}=0 \\
\eta \rightarrow \infty: \quad f^{\prime}=1
\end{gathered}
$$

Note that, when the plate velocity and acceleration tends to zero, the velocity profiles approaches to Hiemenz's Velocity profile, Ref. [1], and when the plate is stationary ( $\dot{S}=0$ and $\ddot{S}=0$ ), Eq. (11) simplifies to the case of Hiemenz flow. In this case, the velocity profile matches Hiemenz velocity profile exactly and this is the only existing reference which can be used for validation.

3.2 Heat Transfer Solution. To transform the energy equation into a nondimensional form for the case of defined wall temperature, we introduce

$$
\theta=\frac{T(\eta)-T_{\infty}}{T_{w}-T_{\infty}}
$$

Making use of transformations (8)-(10), this equation may be written as

$$
\theta^{\prime \prime}+(\tilde{\dot{S}}(t)+\tilde{a}(t) f) \operatorname{Pr} \cdot \theta^{\prime}=0
$$

with the boundary conditions as

$$
\begin{array}{cc}
\eta=0: & \theta=1 \\
\eta \rightarrow \infty: & \theta=0
\end{array}
$$

where $\operatorname{Pr}=\nu / \alpha$, is Prandtl number and prime indicates differentiation with respect to $\eta$. Note that, for $\operatorname{Pr}=1$ and steady-state case the thickness of the fluid boundary layer and thermal boundary layer become the same but in unsteady-state case this concept does not apply and Eq. (18) would not be obtained from substitution of $\theta=f^{\prime}$ in Eq. (11). 


\section{Shear-Stress}

Shear-stress at the wall surface is calculated from

$$
\sigma=\mu \frac{\partial u}{\partial z}{ }_{z=0}
$$

where $\mu$ is the fluid viscosity. Using the transformations (8)-(10), the shear-stress at the flat plate surface becomes

$$
\tilde{\sigma}=\frac{\sigma}{\rho a v}=\xi f^{\prime \prime}
$$

This quantity is presented for different values of $\xi$ in the Presentation of Results section.

\section{Presentation of Results}

As example, the following three distinct velocity functions for constant $A$ are considered:

$$
\dot{S}(t)=A \exp (-t), \quad \dot{S}(t)=A t^{2}, \quad \dot{S}(t)=\operatorname{Sin}(t)
$$

The exponential velocity function above, for example, can be used to model the physical one or two-dimensional solidification problem. Equation (11) for a known plate velocity function is an ordinary differential equation in which the variables $\xi, \dot{S}(t)$, and $\ddot{S}(t)$ are known and can be solved numerically along with Eq. (18) by using a shooting method trial and error and based on the Runge-Kutta algorithm and the results are presented for selected values of $\dot{S}(t)$ and Pr. This procedure is applied for maximum error of less than 0.00001 .

In Figs. 2 and 3, the boundary layer velocity profile has been presented at different values of $\xi$ and for an exponential and polynomial plate velocity functions. As it can be seen, initially the velocity profile with a steep slope approaches the potential flow velocity but as time increases, that is, when the plate velocity and acceleration approaches to zero, the velocity profile gradually tends toward the Hiemenz flow. Therefore, the effect of change of plate velocity is felt more by the boundary layer velocity profile than the effect of plate acceleration. In Fig. 4, one can see the thickness of the boundary layer velocity for sinusoidal plate velocity function. Figure 5 shows velocity in w-direction at selected values of $\xi$ for different time values and exponential plate velocity function. Shear-stress is presented in Fig. 6 for different time and $\xi$ values and for the sinusoidal plate velocity case. The shearstress value on the plate surface becomes constant for large values of $\xi$ at all times. It is interesting to note that the starting point of shear-stress value on $\zeta$-axis is in a fix range for all time values. Figures 7 and 8 show the nondimensional pressure along the

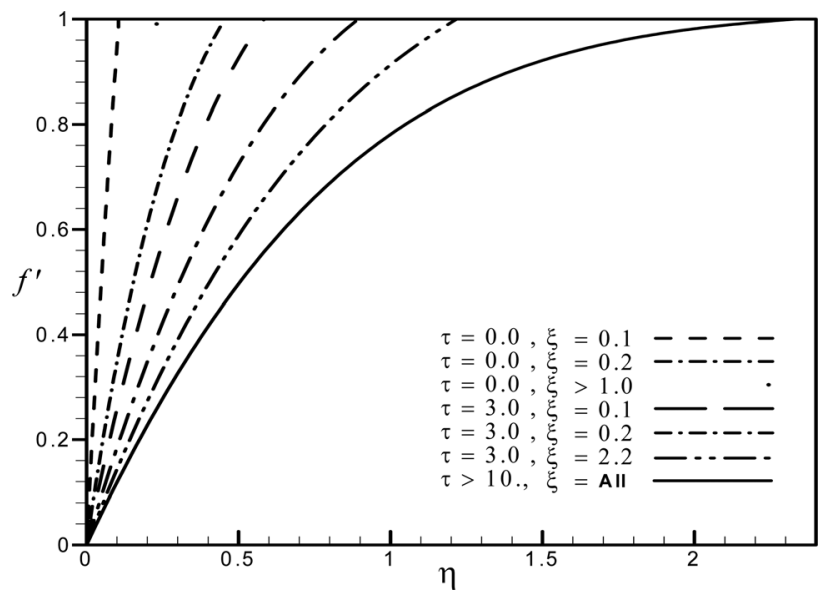

Fig. 2 Velocity in $x$-direction at selected values of $\xi$ for different time values and exponential plate velocity function

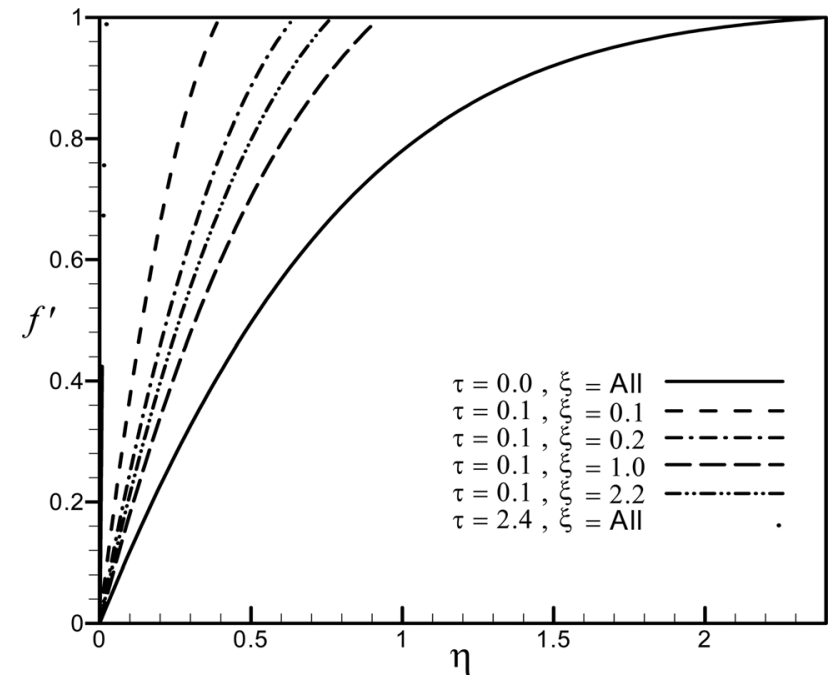

Fig. 3 Velocity in $x$-direction at selected values of $\xi$ for different time values and polynomial plate velocity function

boundary layer thickness for selected values of $\xi$ and different time values. It is interesting to note that this pressure change with respect to $\xi$ is the cause of change of all the quantities like boundary layer thickness, shear-stress, velocity profile, and temperature boundary layer thickness. In Fig. 9, the thermal boundary layer profile for selected values of Prandtl numbers and different values of time are presented for the case of exponential plate velocity function. It can be seen that for large time values, the slope of the thermal boundary layer profile decreases and this shows that the slope of the profile increases sharply with increase of Prandtl number and plate velocity and acceleration toward the impinging flow and sharp increase in heat transfer rate is obvious for the case of the earlier time values and for $\operatorname{Pr}=20$. In Fig. 10, the thermal boundary layer profile is shown for different values of $\xi$ and for exponential plate velocity function. As it is expected, the profile changes are very slight with respect to $\xi$ and that is because the function $f(\eta)$ is the cause of these changes and in higher plate velocity or acceleration and also for higher Prandtl numbers this function diminishes rapidly. Figure 11 depicts the variations of thermal boundary layer thickness with respect to Prandtl number for different values of time and for the case of exponential function of plate velocity. As it is seen from this figure, this thickness

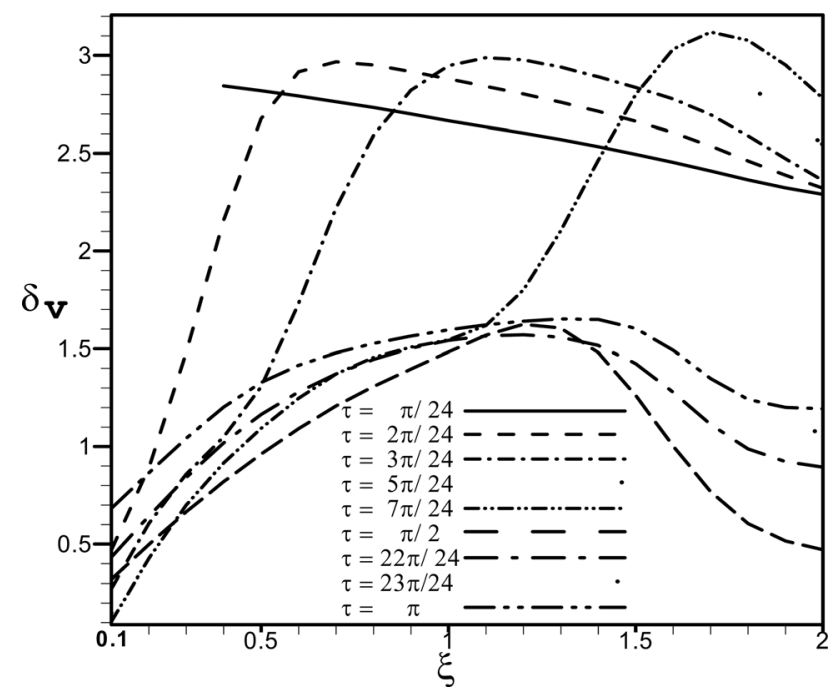

Fig. 4 Boundary layer thickness at different time values and sinusoidal plate velocity function 


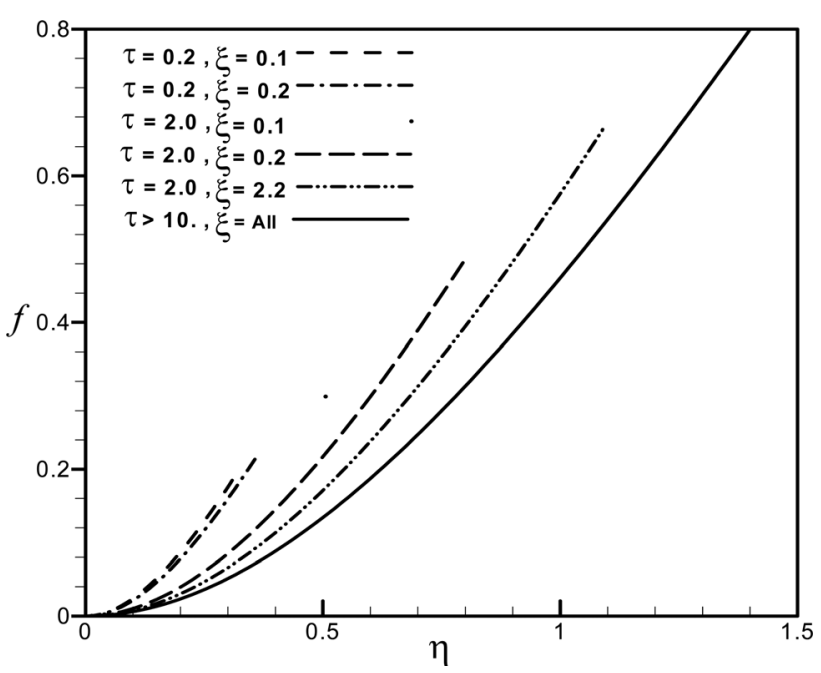

Fig. 5 Velocity in w-direction at selected values of $\xi$ for different time values and exponential plate velocity function

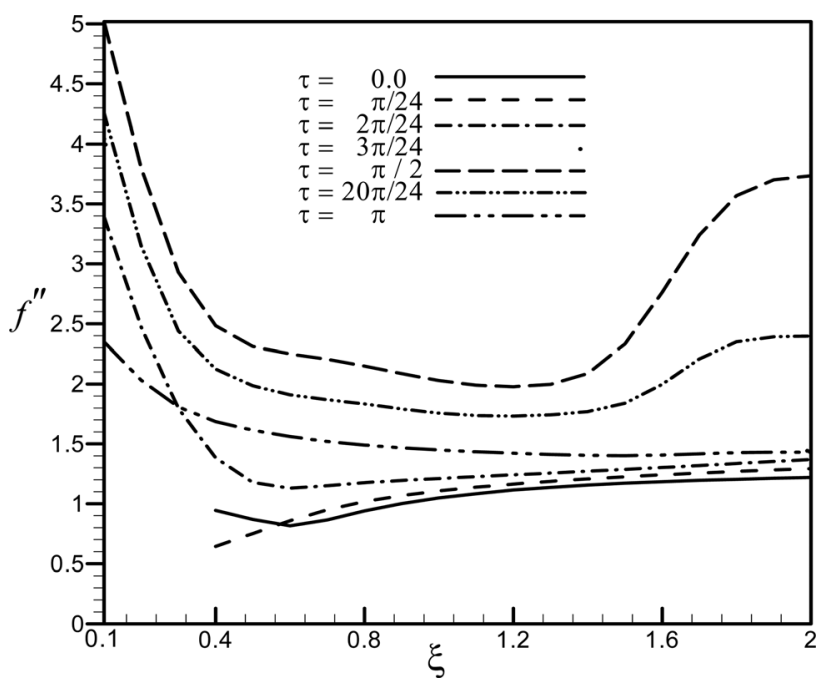

Fig. 6 Shear-stress at different time values and all values of $\xi$ for sinusoidal plate velocity function

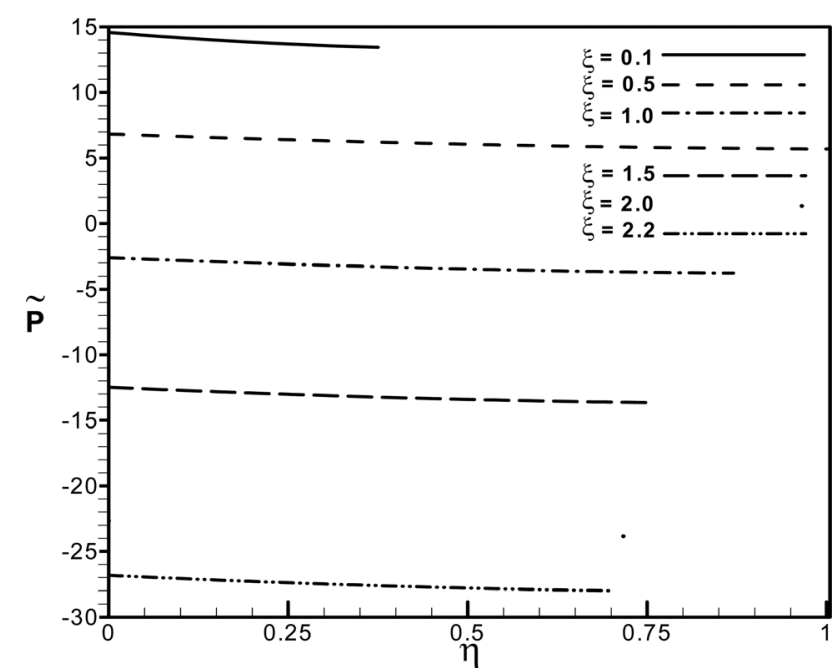

Fig. 7 Pressure variation in boundary layer at certain time and different values of $\xi$ for exponential plate velocity function

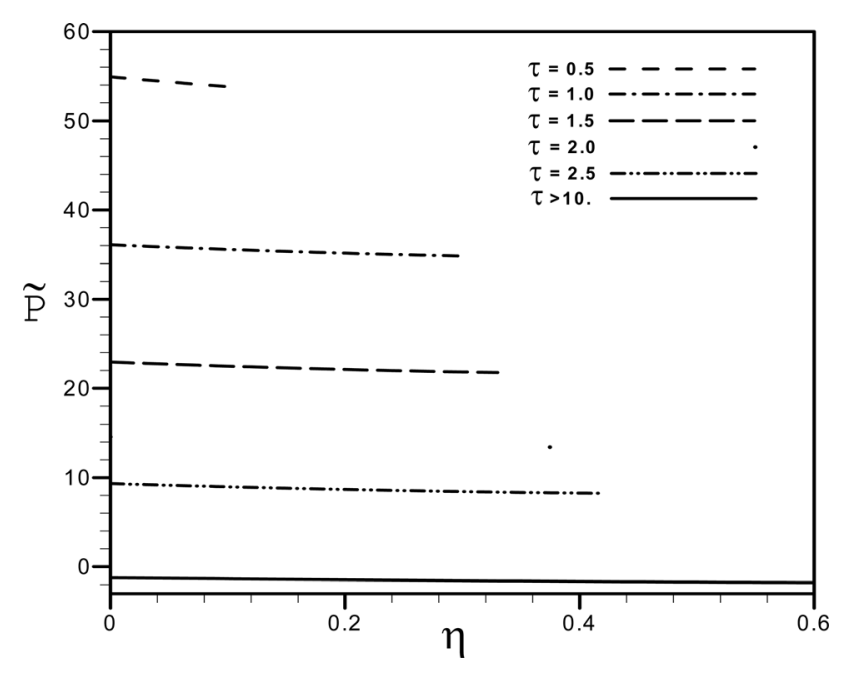

Fig. 8 Pressure variation in boundary layer at $\xi=0.1$ and different values of time for exponential plate velocity function

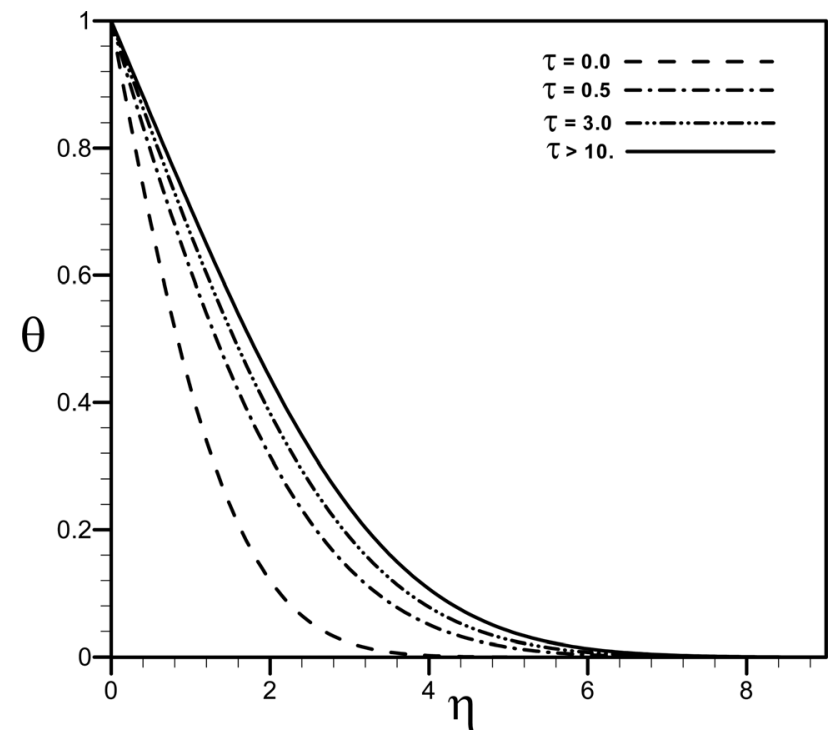

Fig. 9 Thermal boundary layer profile for $\mathrm{Pr}=0.1$ and different time values for exponential plate velocity function

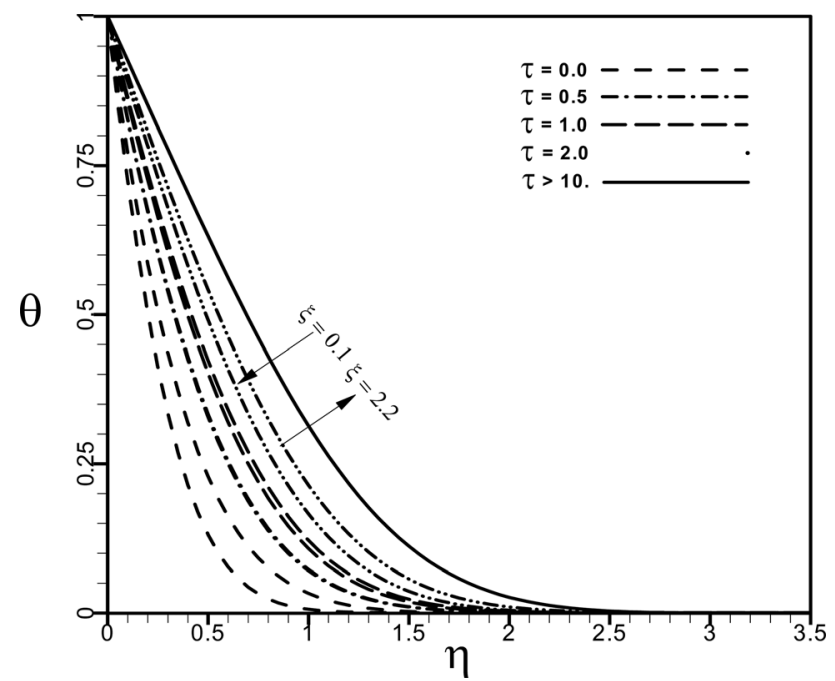

Fig. 10 Thermal boundary layer profile for $\operatorname{Pr}=1.0$ and different time values at $\xi=0.1,2.2$ for exponential plate velocity function

Transactions of the ASME 


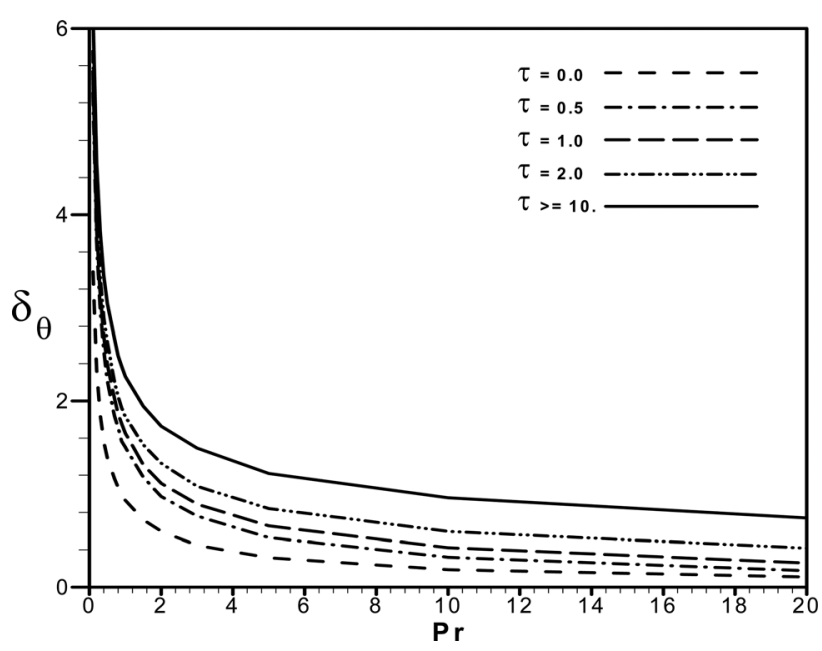

Fig. 11 Thermal boundary layer thickness for different values of time and for all values of Prandtl number and exponential plate velocity function

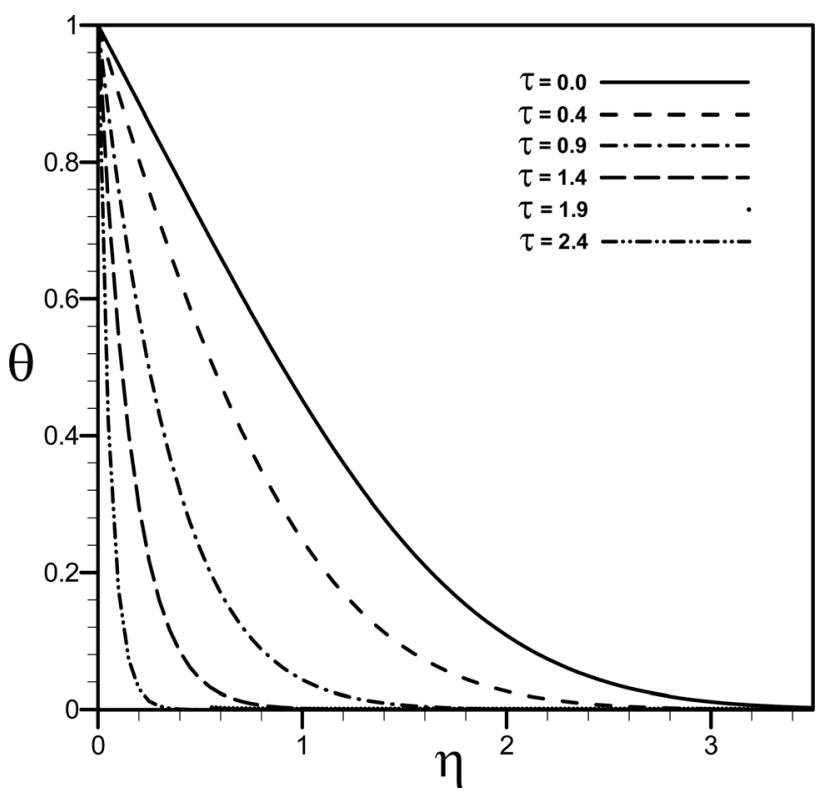

Fig. 12 Thermal boundary layer profile for $\operatorname{Pr}=1.0$ and different time values for polynomial plate velocity function

decreases with the increase of Pr number and increase of plate velocity and acceleration. Figure 12 presents the same trend of changes of thermal boundary layer profile for polynomial and sinusoidal plate velocity plate.

\section{Conclusions}

General formulation and similarity solution of the NavierStokes and energy equations have been derived in the study of two-dimensional unsteady stagnation-point flow and heat transfer impinging on a flat plate where this plate is moving with arbitrary velocity and acceleration functions of time toward main stream or away from it. The results of the stagnation-point flow and heat transfer of the case of stationary plate, Hiemenz flow, is reached by simplifications of this formulation. Presented examples of plate velocity functions are exponential, polynomial and sinusoidal in this paper. All kinds of applications of plate movement is encountered in industry where the stagnation-point flow and heat transfer is involved but our main reason is use of exponential plate velocity function to formulate solidification and melting in these kinds of studies. Velocity and pressure profiles, boundary layer thickness, and surface stress-tensors along with temperature profiles have been presented for different examples of impinging fluid strain rate, selected values of plate velocity, and Prandtl number parameter. The effect of variations of plate velocity is felt more by the boundary layer velocity profile than the effect of plate acceleration. The minimum boundary layer thickness happens at the maximum value of plate velocity and acceleration effect plays a secondary role. When the ratio of nondimensional velocity to the nondimensional acceleration is a linear function, then the boundary layer thickness and shear-stress approach to a constant value as the distance from $z$-axis is increased and this constant value depends on the plate velocity function but when this ratio is a nonlinear function, then the boundary layer thickness and shearstress would not be a constant.

\section{References}

[1] Hiemenz, K., 1911, "Die grenzchicht an einem in den gleichformingen Flussigkeitsstrom eingetauchten geraden KreisZylinder," Dinglers Polytech. J., 326, pp. $321-410$.

[2] Wang, C. Y., 1974, "Axisymmetric Stagnation Flow on a Cylinder," Q. Appl. Math., 32, pp. 207-213.

[3] Gorla, R. S. R., 1976, "Heat Transfer in an Axisymmetric Stagnation Flow on a Cylinder," Appl. Sci. Res., 32, pp. 541-553.

[4] Gorla, R. S. R., 1978, "Nonsimilar Axisymmetric Stagnation Flow on a Moving Cylinder,” Int. J. Eng. Sci., 16, pp. 392-400.

[5] Gorla, R. S. R., 1979, "Unsteady Viscous Flow in the Vicinity of an Axisymmetric Stagnation-Point on a Cylinder,,” Int. Sci. Technol., 17, pp. 87-93.

[6] Cunning, G. M., Davis, A. M. J., and Weidman, P. D., 1998, "Radial Stagnation Flow on a Rotating Cylinder With Uniform Transpiration,” J. Eng. Math., 33, pp. 113-128.

[7] Wang, C. Y., 1973, "Axisymmetric Stagnation Flow Towards a Moving Plate," Am. Inst. Chem. Eng. J., 19(5), pp. 1080-1082.

[8] Grosch, C. E., and Salwen, H., 1982, "Oscillating stagnation-point flow," Proceeding of Royal Society of London, Ser. A, 384, pp. 175-190.

[9] Saleh, R., and Rahimi, A. B., 2004, "Axisymmetric Stagnation-Point Flow and Heat Transfer of a Viscous Fluid on a Moving Cylinder With Time-Dependent Axial Velocity and Uniform Transpiration,” Trans. ASME J. Fluids Eng., 126, pp. 997-1005.

[10] Rahimi, A. B., and Saleh, R., 2007, "Axisymmetric Stagnation-Point Flow and Heat Transfer of a Viscous Fluid on a Rotating Cylinder With Time-Dependent Angular Velocity and Uniform Transpiration," Trans. ASME J. Fluids Eng., 129, pp. 107-115.

[11] Rahimi, A. B., and Saleh, R., 2008, "Similarity Solution of Unaxisymmetric Heat Transfer in Stagnation-Point Flow on a Cylinder with Simultaneous Axial and Rotational Movements," Trans. ASME J. Heat Transfer, 130, p. 054502.

[12] Shokrgozar Abbasi, A., and Rahimi, A. B., 2009, "Non-Axisymmetric ThreeDimensional Stagnation-Point Flow and Heat Transfer on a Flat Plate," Trans. ASME J. Fluids Eng., 131(7), p. 074501.

[13] Shokrgozar Abbasi, A., and Rahimi, A. B., 2009, "Three-Dimensional Stagnation-Point Flow and Heat Transfer on a Flat Plate With Transpiration," J. Thermophys. Heat Transfer, 23(3), pp. 513-521.

[14] Shokrgozar Abbasi, A., and Rahimi, A. B., 2011, "Exact Solution of ThreeDimensional Unsteady Stagnation Flow on a Heated Plate," J. Thermophys. Heat Transfer, 25(1), pp. 55-58.

[15] Bian, X., and Rangel, R. H., 1996, "The Viscous Stagnation-Flow Solidification Problem," Int. J. Heat Mass Transfer, 39, pp. 3581-3594. 